\title{
Estudiantes motivados y participativos. ¿Una utopía? No, una realidad gracias al aula invertida
}

\section{Rocío Martínez Jiménez ${ }^{\mathrm{a}}$ y M. Carmen Ruiz Jiménez ${ }^{\mathrm{b}}$}

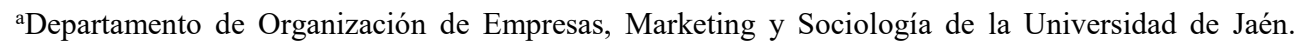
Correo electrónico: rmartine@ujaen.es. ${ }^{b}$ Departamento de Organización de Empresas, Marketing y Sociología de la Universidad de Jaén. Correo electrónico: cruiz@ujaen.es

\begin{abstract}
The flipped classroom is a work methodology that implies a greater students' involvement and commitment to their own learning. They have to adopt a more active and participative role. In this paper, we present the results of an inverted classroom experience in two subjects related to business management. Results show that students considerably improve their motivation and satisfaction with the subject, as well as their degree of attendance and participation in classes. In addition, students acknowledge having worked more on the subject on a daily basis and state that the activities carried out (cases, projects, simulations...) have helped them to better understand and internalize the main concepts of the subject.
\end{abstract}

Keywords: flipped learning, innovation, motivation, participation, business management.

\footnotetext{
Resumen

El flipped classroom es una metodología de trabajo que supone una mayor implicación y compromiso de los estudiantes con su propio aprendizaje, debiendo adoptar un rol más activo y participativo. En este trabajo presentamos los resultados de una experiencia de inversión de aula en dos asignaturas relacionadas con la gestión de empresas. Los resultados muestran que los estudiantes mejoran considerablemente su motivación y satisfacción con la asignatura, así como su grado de asistencia y participación en las clases. Además, los estudiantes reconocen haber trabajado la asignatura más a diario y afirman que las actividades realizadas (casos, proyectos, simulaciones, etc.) les han ayudado a comprender e interiorizar mejor los conceptos fundamentales de la materia.
}

Palabras clave: aula invertida, innovación, motivación, participación, gestión de empresas. 


\section{Introducción}

Si en algo estamos todos de acuerdo es en que nuestros estudiantes no se comportan como lo hacíamos nosotros varios años atrás en esas mismas aulas. Han cambiado las herramientas y las técnicas empleadas en el estudio en el ámbito de la educación superior. En esta era de lo digital, donde prácticamente toda la información o el conocimiento lo tenemos disponible con un click de ratón o con sólo pedírselo a Siri o Cortana, ¿los estudiantes seguirán asistiendo a esas clases magistrales en las que el profesor transmite todo su conocimiento sobre una materia como si sólo él o ella tuviera acceso al mismo? Evidentemente, la respuesta es no. Todos hemos visto estudiantes desmotivados y que dejan de asistir a las clases porque no les aportan ningún valor. Algo así debieron pensar Bergmann y Sams (2012) cuando comenzaron a grabar algunos de los contenidos de sus clases de química para que los estudiantes los visionaran en casa y en el aula se dedicaran a practicar, aplicar e interpretar dichos conceptos. Este fue el principio de una nueva metodología denominada Flipped Classroom, Flipped Learning o, en español, aula o docencia inversa o invertida.

Según este nuevo modelo pedagógico existen dos contextos y momentos de trabajo diferentes: el aula durante la clase; y fuera del aula, antes y después de clase. Este segundo contexto (a diferencia del método tradicional en el que el estudiante hace "los deberes" aplicando lo aprendido en clase) se centra, fundamentalmente, en el visionado de videos o podcasts para introducirse en la parte más conceptual de la asignatura. Por otro lado, el tiempo de clase pasa a dedicarse a la realización de actividades prácticas de trabajo colaborativo o cooperativo como estudios de casos, resolución de problemas, debates, simulaciones, proyectos, etc. que apliquen y refuercen lo aprendido en casa (Khan, 2012; Martínez et al., 2014; Roach, 2014). De esta forma, tal y como plantean Santiago y Bergmann (2018), en las aulas, los estudiantes estarían trabajando fundamentalmente los niveles superiores de la taxonomía de Bloom (ver figura 1).

Figura 1: Taxonomía de Bloom

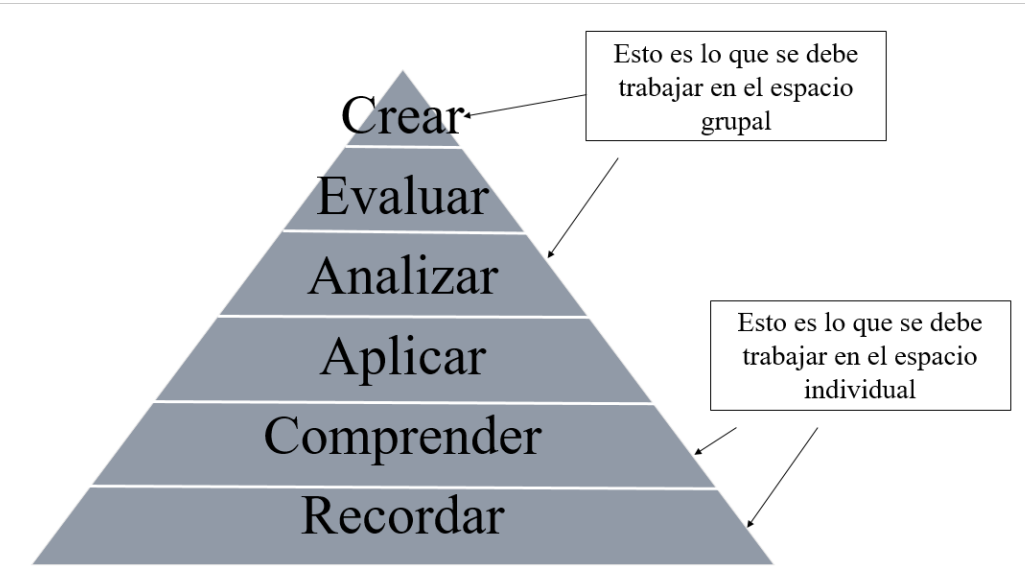

Fuente: Santiago y Bergmann (2018; 105)

En este tipo de enseñanza, los estudiantes, por tanto, deben asumir la responsabilidad de su propio aprendizaje de manera activa, ser más flexibles y adaptarse a esta metodología 
individualizada. Por su parte, el rol del profesor también cambia, dedicándose más tiempo a guiar, orientar y dar retroalimentación a los estudiantes de una manera mucho más individualizada y personalizada que en el sistema tradicional (Tourón y Santiago, 2015).

$\mathrm{Y}$ es que, cuando el estudiante deja de ser un mero receptor de información para pasar a indagar, experimentar, aplicar y descubrir por sí mismo en un ambiente de trabajo dinámico e interactivo, se consiguen mejores resultados (Network, 2018). Así, son varios los trabajos que han mostrado las ventajas o beneficios que tiene la utilización del flipped classroom: mejora del aprendizaje, aumento de la motivación y la autonomía académica (Sidhu et al., 2014); mayor participación de los estudiantes y mejores interacciones con docentes y alumnos, con un mayor feedback hacia los profesores (Bergmann y Sams, 2012; Roach, 2014; Network, 2018; Santiago y Bergmann, 2018); mejora de la satisfacción de los estudiantes con la asignatura (Peña et al., 2018; Aguayo et al., 2019) y mejores resultados académicos de los estudiantes (Szoka, 2013; Walsh, 2013; Esgueva y Carabal, 2018; Montanes et al., 2018).

\section{Objetivos}

Los cambios constantes y la elevada incertidumbre a la que nos enfrentamos diariamente, la nueva cultura digital y sus múltiples oportunidades, el reto de preparar a futuras generaciones para profesiones que aún están por venir... Todo esto demanda un profundo cambio en el papel del profesor cuya función ya no es "transmitir contenidos" sino facilitar procesos individuales de aprendizaje definitivo: enseñar a aprender de forma autónoma durante toda la vida. Por todo lo anterior, el presente trabajo se plantea como principal objetivo mostrar los resultados obtenidos con la experiencia de aula invertida en dos asignaturas relacionadas con la gestión empresarial.

Más concretamente, podemos definir como objetivos específicos los siguientes:

- Conocer la valoración que hacen los estudiantes sobre diferentes aspectos relacionados con esta nueva metodología de enseñanza-aprendizaje.

- Identificar si existen diferencias significativas en dicha valoración según la asignatura, la edad y el sexo de los estudiantes.

- Conocer los principales aspectos positivos y negativos que los estudiantes destacan de esta nueva dinámica en clase.

\section{Desarrollo de la innovación}

\subsection{Contexto}

Esta experiencia de utilización de la metodología de aula invertida ha sido aplicada en dos asignaturas de dos grados diferentes: Gestión de Recursos Humanos en $2^{\circ}$ curso del Grado en Finanzas y Contabilidad (FICO) y Organización y Administración de Empresas en $1^{\circ}$ curso del Grado en Relaciones Laborales y Recursos Humanos (RRLL). Ambas asignaturas 
tienen una carga docente de 6 créditos (con 60 horas de presencialidad) y se han impartido en el primer cuatrimestre del curso 2018-2019.

Se ha trabajado con grupos relativamente reducidos y esto ha facilitado mucho la puesta en práctica de esta herramienta. Concretamente, los estudiantes que han trabajado en el total de estas dos asignaturas han sido 63: 28 en el Grado de FICO y 35 en el Grado de RRLL.

\subsection{Proceso y dinámica de trabajo}

Todo cambio metodológico conlleva, en primer lugar, dedicar un tiempo a la planifición y programación del proceso. De este modo, las profesoras que decidimos invertir nuestras aulas nos reunimos al comienzo del primer cuatrimestre para organizar las asignaturas y la dinámica de clase, trabajando especialmente la selección de recursos y estrategias para la parte grupal (en el aula) sin descuidar en ningún momento la parte más autónoma de trabajo (fuera del aula).

Posteriormente, elaboramos la presentación de las asignaturas para el primer día de clase. Creemos que esto era especialmente importante puesto que el flipped classroom supone un cambio de roles tanto del profesor como de los estudiantes y es conveniente que este aspecto quede claro desde el principio. Ellos deben asumir nuevos compromisos y nuevos roles y deben desarrollar nuevas competencias diferentes a las acostumbradas en el sistema tradicional. Por ello, durante las primeras sesiones de clase les explicamos en qué consiste el aula invertida, para qué nos puede ser útil y cómo la vamos a poner en práctica. Igualmente, presentamos el plan de trabajo y los contenidos y herramientas con los que se iba a trabajar, antes, durante y después de clase. En definitiva, se explicó a los estudiantes el cambio metodológico que se iba a realizar en la asignatura, marcándoles las reglas del juego tanto del espacio individual como del grupal. Igualmente, explicamos que la evaluación de la asignatura sería de tipo formativo y sumativo, esto es, que no sólo se tendría en cuenta el examen teórico final (que representa un $50 \%$ de la calificación) sino que además se valorarían todas las prácticas a realizar $(30 \%)$ y otras actividades como la participación, debates, simulaciones, etc. (20\%), a lo largo del cuatrimestre, recibiendo un feedback continuo. En este punto, nos dimos cuenta de que había varios estudiantes reacios a dicho cambio metodológico y evaluativo, por lo que se les ofreció la posibilidad de elegir metodología; esto es, aquellos alumnos, que por determinadas circunstancias no pudieran o no quisieran seguir esta dinámica de clases, podrían estar tutorizados durante el cuatrimestre y ser evaluados siguiendo el sistema tradicional con un examen final completo teórico-práctico.

A partir de ahí, la dinámica que hemos seguido en cada una de las sesiones de clases ha sido la siguiente:

a) Antes de clase: Para cada unidad, hemos elaborado o les hemos facilitado el acceso a "mini contenidos de formación" en formato multimedia (como videos o presentaciones en power point) y en formato papel (textos, infografías, mapas mentales, etc.).

b) Durante la clase: En clase se ha dedicado entre diez y quince minutos a presentar los objetivos y estructura de cada sesión concreta y a revisar el contenido teórico necesario para el desarrollo de dicha sesión, incidiendo especialmente en las dudas o aspectos que no hubieran quedado claros con el material facilitado. A continuación, se comenzaba con la 
aplicación de las distintas actividades de aprendizaje seleccionadas en función de la adecuación a la temática de la clase (gamificación, enseñanza entre iguales, aprendizaje basado en proyectos, indagación, estudio de casos, debates, resolución de problemas y roleplaying).

c) Después de clase: El tiempo posterior a la clase se ha dedicado a que los alumnos asimilaran los principales conceptos de cada tema.

\subsection{Valoración de la innovación}

Para la valoración de la experiencia de uso de este tipo de metodología, el último día de clase pedimos a los estudiantes que estaban en el aula que completaran un cuestionario voluntario y anónimo que incluía preguntas sobre motivación, grado de participación, percepción del aprendizaje y el rendimiento, etc. usando una escala Likert que iba desde el 1 (muy en desacuerdo) hasta el 5 (muy de acuerdo). Además, les planteamos dos preguntas abiertas para que comentaran lo que consideraban más positivo y más negativo de esta experiencia.

La tasa de respuesta a este cuestionario fue muy alta, puesto que recibimos un total de 58 cuestionarios válidos (un $92.1 \%$ del total). El reparto de dichos cuestionarios entre asignaturas y titulaciones es más o menos equitativo (24 cuestionarios en FICO y 34 en RRLL) y aparece reflejado en porcentajes en el Gráfico 1.

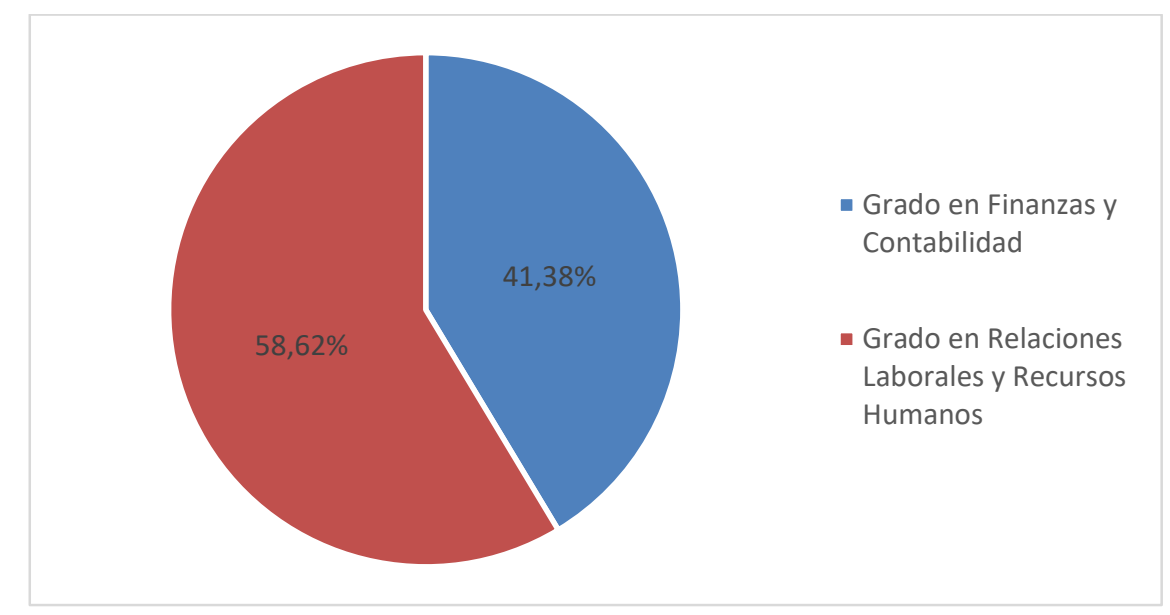

Gráfico 1: Distribución de estudiantes por titulación

Por otra parte, también nos parece adecuado comentar cómo se ha distribuido dicha muestra en cuanto a sexo, también bastante equitativo (ver Gráfico 2) y grupos de edad (ver Gráfico $3)$. En este sentido, los estudiantes, como era de esperar al tratarse de los dos primeros cursos de Grado, se encuentran mayoritariamente por encima de los 18 años y por debajo de los 2223 años. No obstante, también encontramos estudiantes que están por encima de los 24 años siendo la edad máxima la de 35 años. 


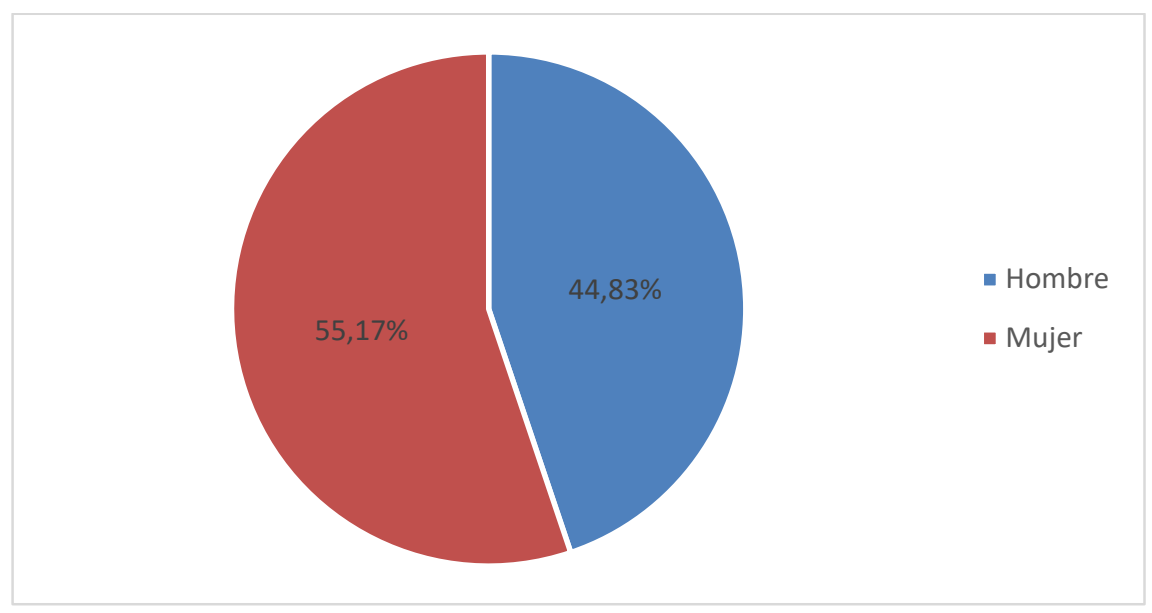

Gráfico 2: Distribución de estudiantes por sexo

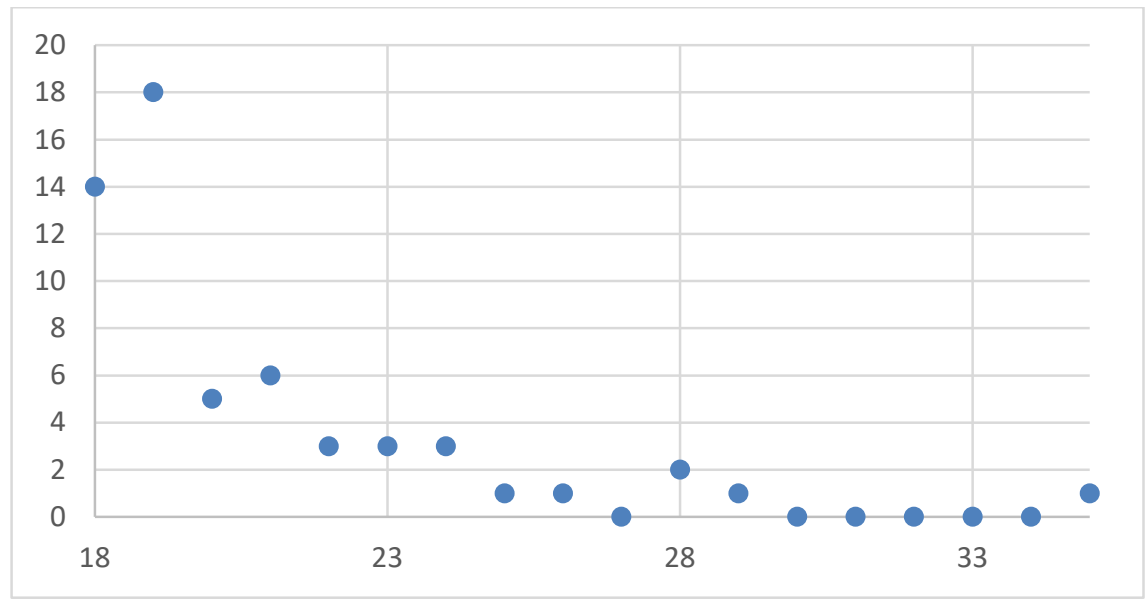

Gráfico 3: Distribución de estudiantes por edad

\section{Resultados}

La primera cuestión que planteamos a nuestros estudiantes era en qué medida el uso de esta nueva metodología había mejorado su motivación por la asignatura. En este caso, la valoración media de todos los estudiantes ha sido de un 4.41 con una desviación típica de 0.676. Por titulaciones, tal y como podemos observar en el Gráfico 4, los estudiantes de FICO son los que han considerado en mayor medida que esta herramienta es motivadora, si bien la valoración es superior en todo momento a 4 , un valor más que satisfactorio. 


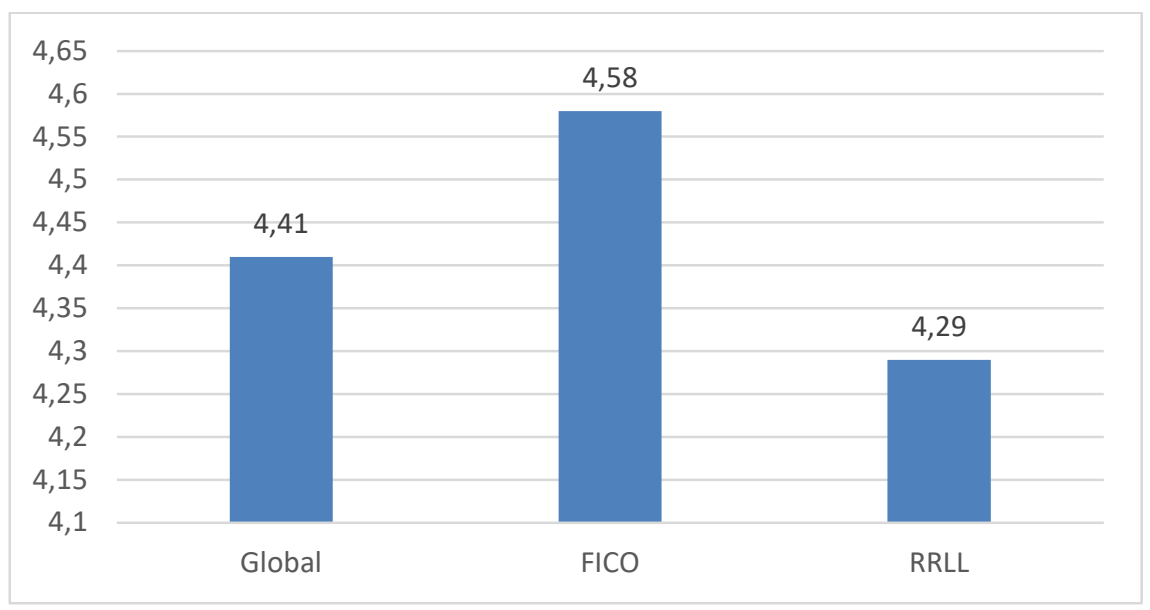

Gráfico 4: Ha mejorado mi motivación por la asignatura

En cuanto a la segunda pregunta, les planteábamos a nuestros estudiantes si la aplicación de esta metodología les había llevado a asistir más a clase de lo que lo hubieran hecho si se hubiera seguido el método tradicional. En este caso, la valoración media también ha superado el 4, con un valor muy elevado (4.69) y una desviación típica de 0.568 . Del mismo modo que antes, tal y como se puede observar en el gráfico 5, los estudiantes de FICO son los que han dado una mayor valoración a este ítem. Este aspecto, lógicamente, fue comprobado por las profesoras a lo largo de todo el cuatrimestre pues observamos que, una vez que los estudiantes "se engancharon" a las primeras actividades, continuaron asistiendo con regularidad a todas las demás clases hasta el último día del cuatrimestre.

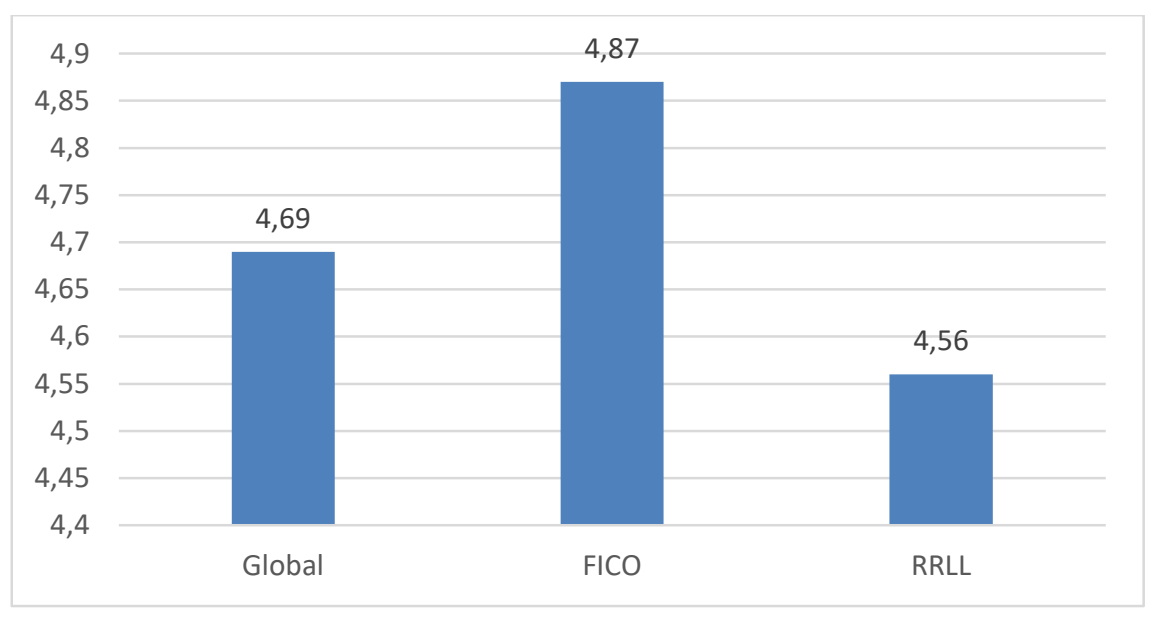

Gráfico 5: Ha aumentado mi asistencia a clase

Otro elemento que hemos valorado ha sido en qué medida trabajar con estos nuevos métodos provoca que los estudiantes trabajen más a diario la asignatura y no lo dejen todo para el final (a unos días del examen). En este caso, la valoración también es positiva, aunque un poco más baja, con una media de 4.22 (desviación típica de 0.918 ). Por titulaciones, tal y como presentamos en el gráfico 6 , vuelven a repetirse los patrones que hemos comprobado hasta ahora, con FICO con la mayor valoración. 


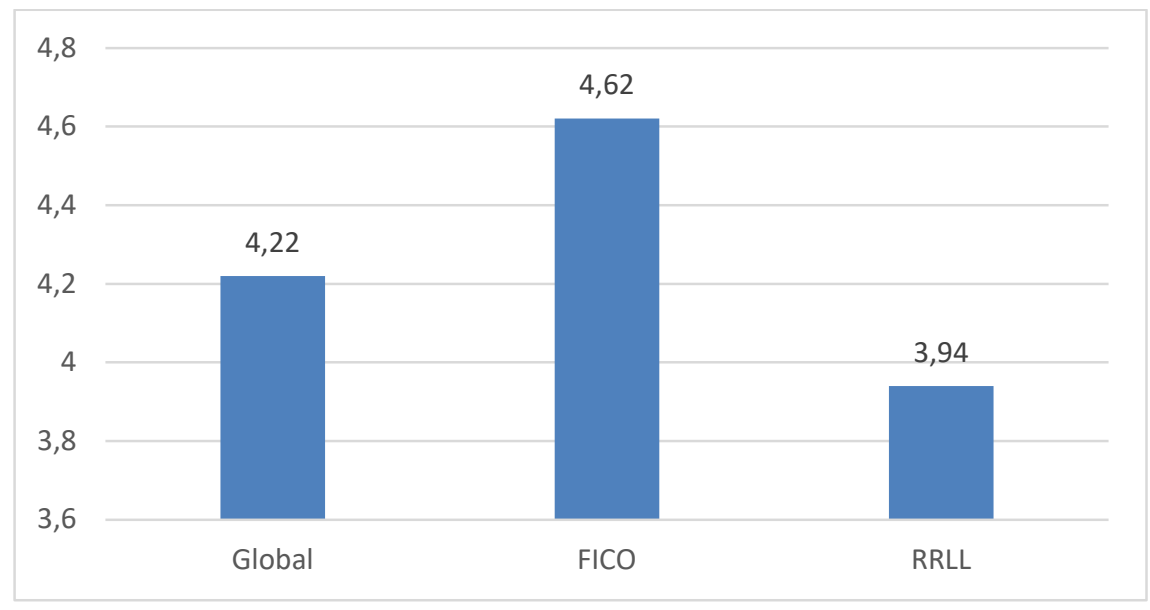

Gráfico 6: Ha hecho que trabaje más a diario la asignatura

El rol más activo y participativo que tenían los estudiantes en su propio proceso de aprendizaje también ha sido valorado en el cuestionario. En este caso, la valoración media es igualmente alta (4.34, con una desviación típica de 0.828$)$. Por titulaciones, comprobamos en el gráfico 7 que se sigue repitiendo la valoración más alta para los estudiantes de FICO.

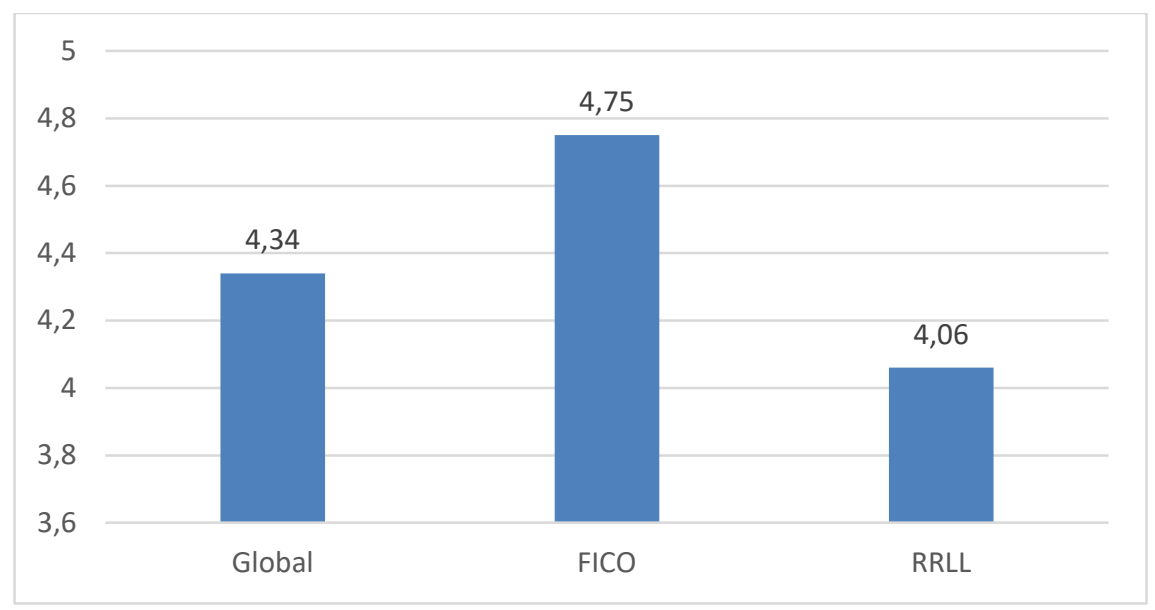

Gráfico 7: Ha generado un aprendizaje más activo y participativo

Del mismo modo, quisimos saber en qué medida los estudiantes consideran que este sistema mejoraría sus calificaciones finales (rendimiento) en la asignatura. Este ítem puntúa un poco más bajo que los anteriores (3.76, con una mayor desviación típica de 1.537). Por titulaciones, nuevamente son los estudiantes de FICO los que puntúan más elevado (ver gráfico 8). 


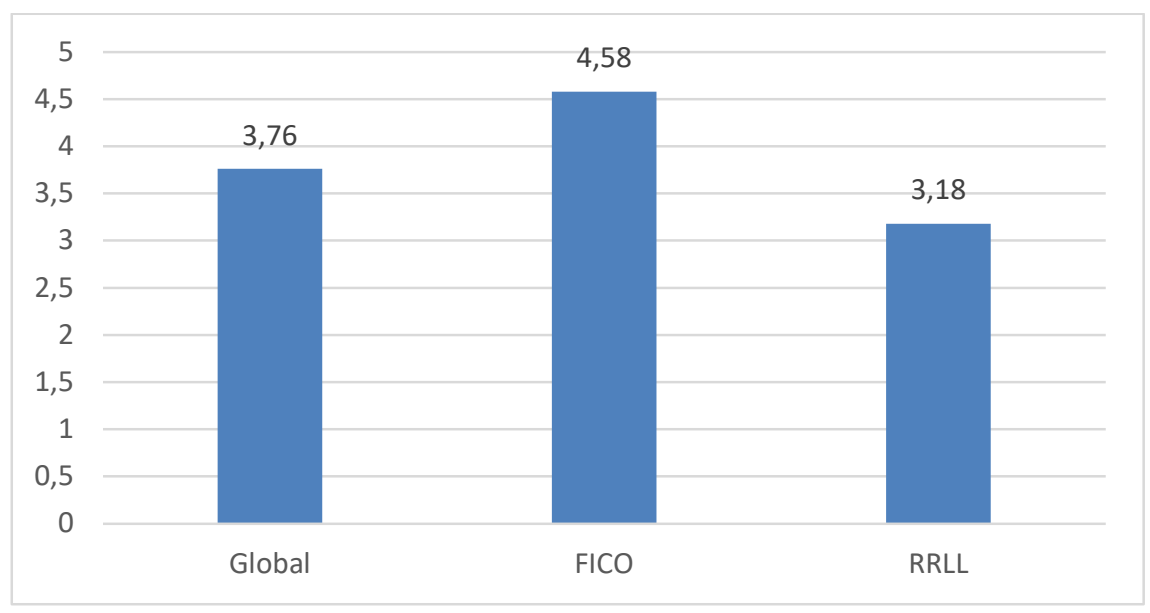

Gráfico 8: Creo que mejorará mi rendimiento (calificación final) en la asignatura

Por otra parte, creíamos que el hecho de utilizar esta metodología podía suponer para ellos un esfuerzo excesivo o una carga de trabajo mucho más elevada de la que están acostumbrados. Por este motivo, quisimos plantearles esta cuestión esperando en este caso que las valoraciones estuvieran por debajo del 3, lo que significaría que la carga no había sido excesiva (aunque sí fuera mayor que en otras asignaturas). $\mathrm{Y}$ así ha sucedido, pues los estudiantes han otorgado una valoración media de 2.76 a este aspecto (desviación típica de 0.904). Por titulaciones, tal y como podemos observar en el gráfico 9 , los estudiantes de RRLL han dado una mayor puntuación a este aspecto, esto es, consideran en mayor medida que ha habido más carga de trabajo, pero sin llegar a considerarla extremadamente excesiva.

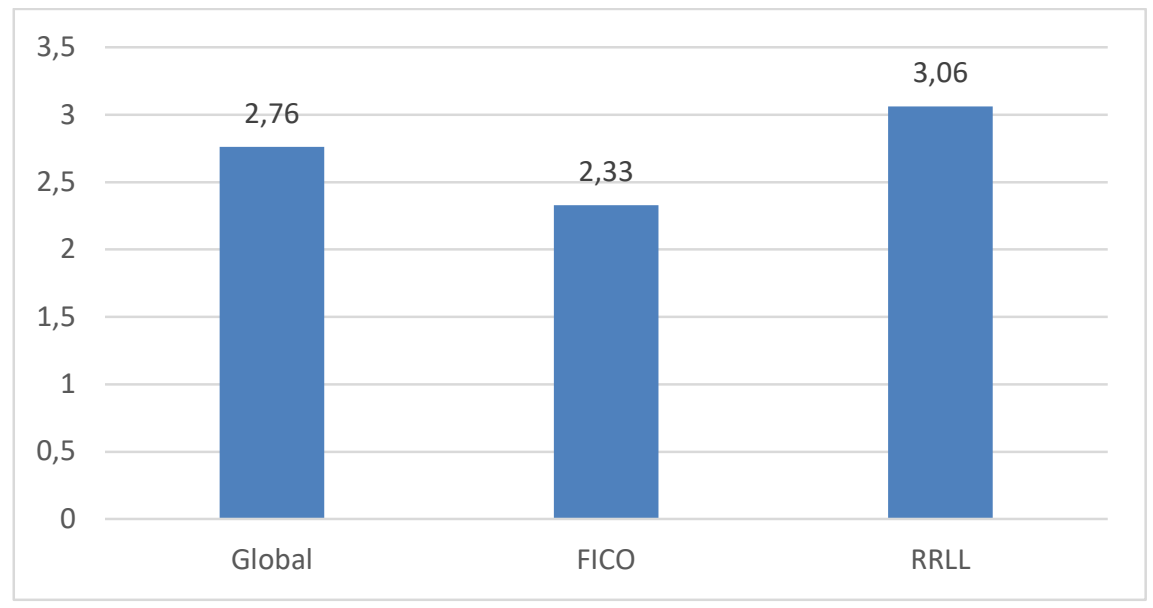

Gráfico 9: Ha supuesto una carga excesiva de trabajo en el cuatrimestre

Por último, quisimos analizar el grado de satisfacción general con esta nueva metodología. La valoración media obtenida en esta pregunta es muy elevada y satisfactoria, con un valor de 4.81 (con una desviación de 0.395). Por titulaciones, como se observa en el gráfico 10, es en FICO nuevamente donde se alcanza una mayor valoración. 


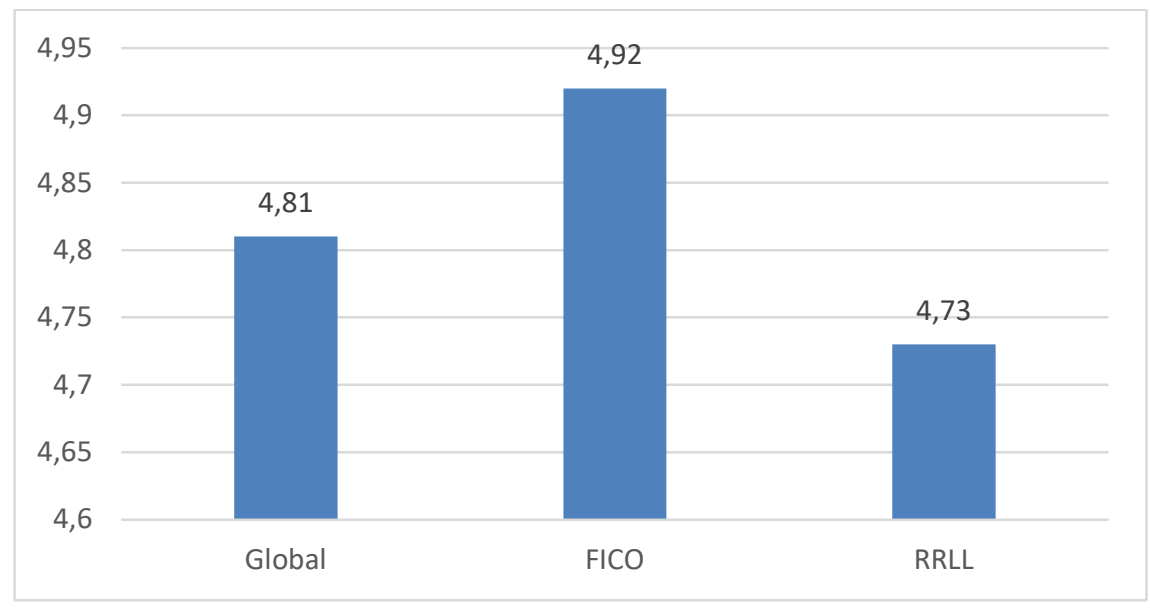

Gráfico 10: Grado de satisfacción con la metodología

En segundo lugar, quisimos analizar si existen diferencias significativas entre los grupos de titulaciones mediante un estudio de tablas de contingencia (con Chi-cuadrado) y un estudio ANOVA. En la tabla 1 podemos comprobar como las diferencias sí son estadísticamente

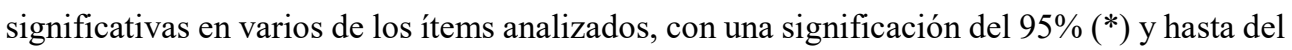
$99 \%$ (**) en algunos casos. Y es que, tal y como hemos ido comprobando en los análisis anteriores, los estudiantes de FICO, en general, son los que han realizado una mejor valoración de esta experiencia de inversión de aula.

Tabla 1: ANOVA y Chi-cuadrado por Titulación

\begin{tabular}{lcc}
\hline & $\begin{array}{c}\text { ANOVA } \\
\text { p-value }\end{array}$ & $\begin{array}{c}\text { Chi-cuadrado de Pearson } \\
\text { Sig, asintótica }\end{array}$ \\
\hline Motivación & 0.109 & 0.332 \\
Asistencia & $0.036^{*}$ & 0.109 \\
Trabajo diario & $0.004^{* *}$ & $0.015^{*}$ \\
Aprendizaje activo y participativo & $0.001^{* *}$ & $0.001^{* *}$ \\
Mejora de rendimiento & $0.000^{* *}$ & $0.001^{* *}$ \\
Excesiva carga & $0.002^{* *}$ & $0.033^{*}$ \\
Satisfacción & 0.085 & 0.083 \\
\hline
\end{tabular}

También hemos realizado este análisis, pero considerando la edad y el sexo como factores. Sin embargo, en ambos casos hemos de decir que las diferencias han resultado no ser estadísticamente significativas, con p-values siempre superiores a 0.05 . De esta forma, ni la edad ni el sexo de los estudiantes parecen tener influencia en una mejor o peor valoración de la experiencia de aula invertida.

Finalmente, para logar nuestro tercer objetivo, queremos presentar los resultados del análisis cualitativo realizado a las dos preguntas abiertas que inclúamos en el cuestionario (los aspectos positivos y negativos que, a su juicio, tiene esta metodología de trabajo). Así, sobre lo positivo o lo que más les ha gustado de la experiencia de aula invertida, la respuesta más 
repetida ha sido el hecho de trabajar en equipo con los compañeros, lo que hace que se mejoren las relaciones entre ellos. A continuación, otro aspecto también muy repetido ha sido que las clases son más entretenidas, amenas y dinámicas. Igualmente, ha sido muy valorado el hecho de realizar muchas prácticas que ellos ven reales y aplicadas y que les sirven para aprender más y mejor.

Otras cuestiones valoradas positivamente, aunque con menor frecuencia, tienen que ver con la mejora de la motivación y el interés por la asignatura así como la mayor participación en clase; el trabajar la asignatura al día y no dejarlo todo para el final; el hecho de que la evaluación tenga en cuenta más aspectos que no sólo la memorización de conceptos y que vayan sumando puntos para la nota final y, finalmente, la mejora de la relación con la profesora que muestra interés y preocupación por el aprendizaje de los estudiantes.

Sobre lo negativo, hay más unanimidad y se suelen repetir básicamente tres respuestas: que es un método que requiere mucho trabajo continuado y asistencia diaria a clase; aquellos estudiantes que son más tímidos y les cuesta más hablar en público muestran más reticencia a las exposiciones; y, finalmente, la gran mayoría de personas ha hecho referencia a la dificultad que supone el trabajo en equipo: compañeros que no se implican lo suficiente o con los que no se llevan especialmente bien, dificultad de coordinación fuera del aula para poder trabajar a la vez, etc.

\section{Conclusiones}

Invertir el aula ha sido una experiencia muy gratificante en las dos asignaturas en las que se ha llevado a cabo tanto para los estudiantes como para las profesoras implicadas. En general, podemos decir que se ha mejorado mucho el clima de trabajo en clase. Ambas profesoras veníamos de cursos en los que habíamos vivido una gran desmotivación de los estudiantes en clase, incluso con problemas de actitud muy negativa, y un continuado pasotismo por su parte que les llevaba a dejar de asistir a las aulas en la mayoría de las ocasiones para "prepararse la asignatura por su cuenta".

Con esta nueva metodología se ha mejorado de una manera importante la dimensión afectivaemocional tanto del profesorado como del alumnado que ha aumentado su interés, motivación y participación en la asignatura hasta el último día de clase.

Las relaciones tanto entre los propios compañeros como entre los estudiantes y la profesora han sido mucho más fluidas y cercanas, identificando ellos que las profesoras no sólo se dedicaban a explicar, sino que, especialmente, se preocupaban porque aprendieran y comprendieran la asignatura, ayudándoles individualmente en el caso de que en algo se quedaran un poco retrasados. Esto muestra que este sistema tiene, como una de sus principales fortalezas, la identificación de la diferenciación entre los estudiantes. No todos son iguales ni aprenden al mismo ritmo, pero el tradicional sistema de lección magistral y estudio en casa no permite al profesorado detectar estas individualidades. Sin embargo, al invertir el aula, el profesorado puede ayudar a esos estudiantes que pueden tener unas necesidades educativas especiales o que, simplemente, necesitan más tiempo o más 
explicación para comprender e interiorizar la materia. En este sentido, también hemos podido comprobar que los estudiantes más tímidos o reservados se encuentran más a gusto trabajando en los grupos pequeños donde no tienen tanto reparo para aportar sus ideas como les sucede en el grupo grande.

Además, la realización de diversas actividades (casos, simulaciones, dinámicas en grupo, gamificación, etc.) ha ayudado a nuestros estudiantes a aprender y comprender mejor la materia, a ver su aplicación real en el mundo empresarial y, sobre todo, a trabajar la asignatura prácticamente al día y no dejándolo todo para el final. Al hacer esto, ellos mismos han sido conscientes de que su rendimiento o calificación en la asignatura sería mejor; no sólo por el hecho de que estaban asentando mejor los conceptos al aplicarlos y entenderlos, sino porque se estaban teniendo en cuenta otros aspectos más allá de la mera retención memorística de contenidos.

En este sentido, sin ellos ser demasiado conscientes, se estaban trabajando las diferentes competencias que se incluían en la guía docente y es por ello que las profesoras iban valorando y otorgando una puntuación a esas otras actividades que realizaban. En este sentido, queremos hacer una especial mención a la competencia de "trabajo en equipo". Y es que, curiosamente, esto ha sido una de las cosas que los estudiantes han valorado como más positivo de la experiencia (el trabajar con los compañeros de manera habitual) pero que también ha generado las mayores críticas o comentarios negativos sobre el método. Ellos se han dado cuenta de lo difícil que es trabajar en equipo, con personas que no conoces, que no trabajan siempre con la misma energía, el mismo interés o las mismas ganas que le ponen otros, que algunas veces no se comprometen lo suficiente con la tarea a realizar, etc. Así, por ejemplo, era curioso ver cómo algunas personas preferían que los grupos siempre fueran los mismos (aquellos en los que sí había una buena coordinación y cohesión como equipo) mientras que otros preferían que se rotaran y se hicieran al azar (aquellos donde había surgido algún roce o conflicto). Esta es quizás una de las dificultades a las que se enfrenta el profesorado que quiera trabajar este tipo de metodología en clase.

Otro inconveniente de este método es que los estudiantes consideran que, en ocasiones, la carga de trabajo puede llegar a ser algo excesiva. En este sentido, el profesorado debe ser cauto a la hora de calcular el tiempo de trabajo autónomo que puede suponer fuera del aula para el estudiante de tal forma que no tenga esa sensación de demasiado esfuerzo. Como todo, la inversión del aula es un proceso en el que año tras año se deben ir corrigiendo aquellos aspectos que pueden no funcionar correctamente y creemos que este es uno de ellos. Además, en nuestro caso, hemos tenido la suerte (o la desgracia) de ser la única asignatura del mismo cuatrimestre que seguía este tipo de metodología, pero pensamos que, si esto se ampliara al resto de asignaturas, se necesitaría una buena coordinación entre el profesorado de dichas asignaturas para evitar que, en un determinado momento de tiempo, los estudiantes se encontraran con una acumulación excesiva de trabajo fuera del aula.

Por último, como ya dijimos al explicar el desarrollo de la innovación, creemos que esta experiencia ha sido tan fructífera debido al hecho de que hemos tenido pocos estudiantes en cada asignatura. Si hubiera que aplicar esta metodología en aulas donde el número de 
matriculados fuera superior, estamos convencidas de que no hubiera funcionado de la misma manera ni se habrían obtenido los mismos resultados.

Como limitación de este trabajo, sabemos que se trata de una experiencia con pocos estudiantes implicados lo que dificulta la generalización de resultados y que debería ser replicada con otras asignaturas y en otros grados. Igualmente, como otra línea futura de trabajo, proponemos el análisis de las calificaciones obtenidas en la asignatura comparando este curso académico 2018-2019 con el curso anterior en el que no se empleó esta metodología, para ver si ha habido o no una mejora de los resultados de los estudiantes.

\section{Referencias}

AGUAYO VERGARA, M.; BRAVO MOLINA, M.; NOCETTI DE LA BARRA, A.; CONCHA SARABIA, L. y ABURTO GODOY, R. (2019). "Perspectiva estudiantil del modelo pedagógico flipped clasroom o aula invertida en el aprendizaje del inglés como lengua extranjera” en Revista Educación, 43(1). https://doi.org/10.15517/revedu.v43i1.31529

BERGMANN, J. y SAMS, A. (2012). Flip your classroom: Reach every student in every class every day. Washington, DC: International Society for Technology in Education.

ESGUEVA LÓPEZ, V. y CARABAL MONTAGUD, M.A. (2018). "Innovación docente: los alumnos diseñan un manual cooperativo mediante el flipclass”. En Congreso IN-RED 2018, Valencia.

KHAN, S. (2012). The one world schoolhouse: Education reimagined. London: Hodder and Stoughton.

MARTÍNEZ, W.; ESQUIVEL, I. y CASTILLO, J. (2014). “Aula invertida o modelo invertido de aprendizaje: Origen, sustento e implicaciones” en Esquivel, I. (Coord.). Los modelos tecno-educativos, revolucionando el aprendizaje del siglo XXI. México (143-160).

MONTANES, N.; SÁNCHEZ-NACHER, L.; FENOLLAR, O.; GARCÍA-SANOGUERA, D. y QUILES CARRILLO, L. (2018). ¿Por qué es importante aplicar Docencia Inversa? ¿Cómo hacerlo?” En Congreso IN-RED 2018, Valencia.

NETWORK, F.L. (2018). "What is Flipped Classroom". <https://www.theflippedclassroom.es/whatis-innovacion-educativa/> [Consulta: 15 de febrero de 2019]

PEÑA, B.; ZABALZA, I.; LLERA, E.; USÓN, S. y ROMEO, L.M. (2018). “Aplicación del modelo de clase inversa en el campo de la Ingeniería Térmica: análisis y comparación de varios contextos”. En Congreso IN-RED 2018, Valencia.

ROACH, T. (2014). "Student perceptions toward flipped learning: New methods to increase interaction and active learning in economics" en International Review of Economics Education, 17, 74-84.

SIDHY, D.; KWAN, R.; PEXMAN, P. y SIAKALUK, P. (2014). "Effects of relative embodiment in lexical and semantic processing of verbs" en Acta psychologica, 149, 32-39.

TOURÓN, J. y SANTIAGO, R. (2015). "El modelo Flipped Learning y el desarrollo del talento en la escuela" en Revista de Educación, 368, 196-231.

SANTIAGO, R. y BERGMANN, J. (2018). Aprender al revés. Flipped learning 3.0 y metodologías activas en el aula. Barcelona: Paidós Educación. 
SZOKA, J. (2013). Measured results demonstrate enhanced learning outcomes in the flipped classroom. < <ttps://www.emergingedtech.com/2013/05/measured-results-demonstrate-enhancedlearning-outcomes-in-the-flipped-classroom/> [Consulta: 15 de febrero de 2019]

WALSH, K. (2013). Gathering evidence that Flipping the classroom can enhance learning outcomes. $<$ https://www.emergingedtech.com/2013/03/gathering-evidence-that-flipping-the-classroom-canenhance-learning-outcomes/> [Consulta: 15 de febrero de 2019] 